WOODS, P. (1979). The Divided School, London: Routledge and Kegan Paul.

YOUNG, T.R. and BEARDSLEY, P. (1968). "The Sociology of Classroom Teaching: A MicroFunctional Analysis", Journal of Educational Thought, Vol. 2, No. 3, pp. 175-186. 


\section{LA RELACION ENTRE LOS CODIGOS SOCIOLINGUISTICOS Y LOS CODIGOS EDUCATIVOS (1977)}

Quisiera presentar juntas las relaciones más importantes entre los ensayos del Volumen 1 y los presentados en el volumen 3. El bosquejo de la unión fue dado en el artículo titulado "A brief account of the theory of codes" que se publicó en la segunda edición del Volumen 1. Sin embargo, el asunto fue necesariamente limitado ya que no podía esperar que el lector hubiera tenido acceso a los ensayos de este volumen (Vol. 3). La tesis general se compone de dos vertientes ínter-relacionadas:

1) Cómo las relaciones de clases regulan la estructura de la comunicación en la familia y por lo tanto, la orientación del código sociolingüístico inicial de los niños.

2) Cómo las relaciones de clase regulan la institucionalización de los códigos elaborados en la escuela, las formas de su transmisión y por lo tanto las formas de su manifestación.

Ahora bien, la segunda tesis a menudo es ignorada por aquellos que evalúan la investigación; sin embargo, el volumen 3, muestra que ha habido una serie de artículos que desde 1964, han intentado el análisis de la educación como una agencia de control simbólico. Existen interacciones cruciales entre la tesis 1 y la tesis 2 . La manifestación de los códigos elaborados transmitidos por la familia son a su vez regulados por la forma de su transmisión en la escuela. Los presupuestos de clase de los códigos elaborados se encuentran en la clasificación y enmarcación del conocimiento educativo y en la ideología que dicha clasificación y enmarcamiento expresan.

Es conveniente distinguir analíticamente los tres niveles de la tesis general. (Véase figura 1). El nivel 1 se refiere al nivel macro-institucional. El nivel 2 se refiere al nivel de transmisión, esto es, a cualquier agencia de transmisión, como p.e. la familia, el grupo pre-escolar, la escuela secundaria ${ }^{* *}$. El nivel 3 se refiere al nivel textual, y más específicamente a la forma de las relaciones sociales específicas del nivel 2 y se centra fundamentalmente en la selección de los significados que activan la codificación lingüística. En este nivel, nos preguntamos qué reglas profundas subyacen a la producción del texto $^{* * *}$. En el nivel 2 la unidad mayor de análisis es una agencia específica y en el nivel 3 la unidad mayor de análisis es un texto específico. Así, en principio, podríamos movernos desde los rasgos distintivos de un texto específico (nivel 3) hacia los rasgos distintivos macro-institucionales (nivel 1). Digo "en principio" porque no conozco ninguna teoría que nos capacite para efectuar esta progresión más que en términos extraordinariamente generales: una de las dificultades consiste en que las teorías que son relativamente fuertes en el nivel macro-institucional son muy débiles en el análisis del proceso de transmisión mientras que teorías que son muy fuertes en el análisis de este último son muy débiles en el análisis del primero. La teoría de los volúmenes uno y tres se centra en los principios que subyacen a los procesos de interiorización y exteriorización, sin embargo el enfoque debe ser tal que las relaciones sociales sobre las cuales este proceso descansa no sean abstraídas de una situación institucional y cultural más amplia. Este problema a menudo se conceptualiza como el problema de las relaciones entre lo

\footnotetext{
* Este artículo integra un extracto de la introducción al Volumen III y la Nota A del Capítulo 8, Vol. 3. Traducido con permiso del autor por Mario Díaz.

** El desarrollo de la tesis produce más tarde un nivel de recontextualización entre el nivel 1 (nivel generativo) y el nivel 3 (nivel de transmisión). Véase Díaz (1983) y Bernstein y Díaz (1984). N.T.

${ }_{* * *}^{*}$ El texto para Bernstein es la forma de la relación social hecha visible, material, palpable; el texto es la transformación de prácticas de interacción especializadas. (Véase, “Códigos, Modalidades...”, Pág. 6) N.T.
} 
macro y lo mi-cro o entre la macro-sociología y la micro-sociología (ningún sociólogo llamaría a esta última, psicología social). Sin embargo, esta clase de distinción es en sí misma el producto de una clasificación y de una enmarcación fuertes dentro de la división social del trabajo del conocimiento sociológico. Este fuerte aislamiento hace extraordinariamente difícil entender cómo las contradicciones, rupturas, ambigüedades y dilemas se constituyen en el individuo. Es casi, como si existiera un principio de autoderrota en el corazón de la actividad sociológica, que encuentra su expresión en términos de distinciones tales como macro y micro y sustancialmente en términos de las polémicas dentro de o entre estos niveles. Los aspectos interrelacionados de la tesis se presentan en la siguiente figura.

Nivel I

Macro-institucional Generativo

Nivel 2

(Transmisión) (Controles)

Clasificación Enmarcaciones

Nivel 3 (Textual)
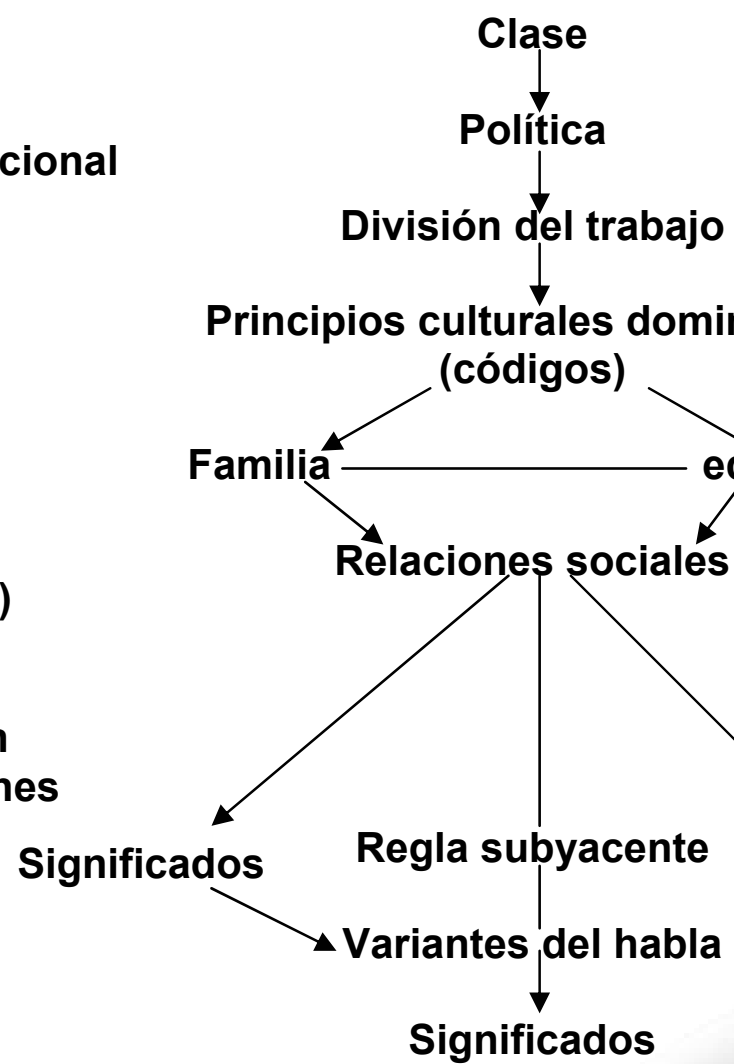

Principios culturales dominantes
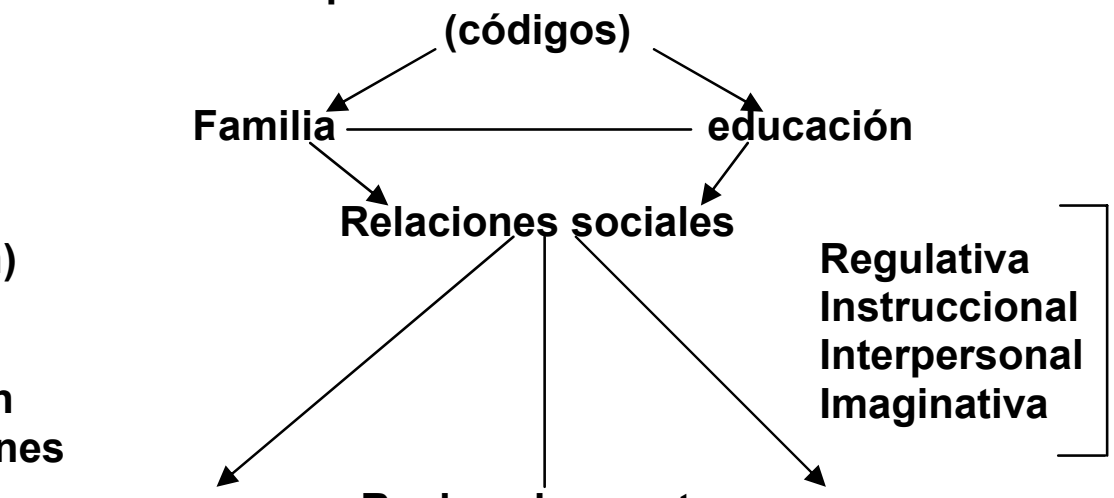

Significados

Contextos códigos

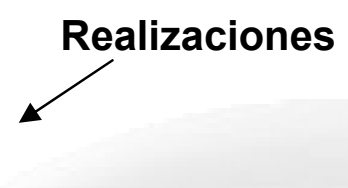

Contexto independiente (significados universalistas)

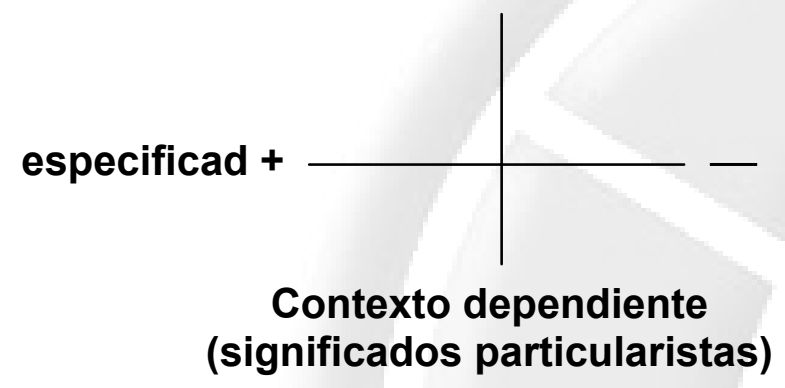

Nivel 1 - Macroinstitucional (generación)

Las relaciones de clase actúan fundamentalmente sobre la división del trabajo mediante la estructuración de sus bases morales, esto es, mediante la creación de relaciones subyacentes de producción, distribución y consumo. Las relaciones de clase 
regulan la transmisión, participación, y la posibilidad de cambio de las categorías culturales dominantes. La tesis se enfoca sobre las consecuencias de lo anterior para sólo dos agencias de preparación, la familia y la escuela. La tesis examina más específicamente la adquisición de los principios de codificación en la familia y en la escuela, los cuales, se considera, configuran la conciencia. Tal configuración, sin embargo, contiene las rupturas y contradicciones que son inherentes a los rasgos específicos de las relaciones de clase. Así, el Nivel 1 se refiere a los orígenes y distribución de los principios de interpretación dominante (códigos) en la medida en que éstos son iniciados y mantenidos por la familia y la educación. Diríamos que el estudio de este nivel es más un programa que algo que existe concretamente.

\section{Nivel II - Transmisión}

Este aspecto se refiere al análisis sustancial de la estructura de las agencias de transmisión entre las cuales la familia y la educación juegan un papel crucial. Aquí, la proposición básica consiste en que la estructura de las relaciones sociales determina los principios de comunicación y de esta manera configura las formas de conciencia. De esto, se deduce que el cambio en la estructura de las relaciones sociales puede cambiar los principios de la comunicación y de esta manera las formas de conciencia. En términos más específicos, la forma de la relación social transforma la semántica potencial en una semántica específica que activa la forma de su manifestación y las condiciones para la manifestación de un patrón en lugar de otro. Los conceptos usados para el análisis de las agencias de transmisión son el de clasificación y el de enmarcación. El argumento, en este caso, consiste en que en la medida en que la clasificación y la enmarcación dentro y entre las agencias de transmisión cambia, también cambian las bases sociales de la comunicación y de su manifestación. Es importante añadir que aquí nos referimos no sólo a las relaciones entre la familia y la educación sino también al trabajo y al descanso. Con el propósito de operacionalizar la tesis en el nivel de transmisión podemos distinguir analíticamente cuatro situaciones cruciales para su exploración: la regulativa, la instruccional, la imaginativa y la inter-personal (Véase "Clases Sociales, lenguaje y socialización" en este volumen). Así, si se estuviera estudiando la familia empíricamente, uno podría examinar, al menos, la clasificación y el enmarcamiento de estas cuatro situaciones y las variantes del habla que cada situación produce. Puesto que la clasificación se define en términos de la fuerza de los aislamientos, ésta necesariamente apunta hacia la jerarquía y hacia sus manifestaciones simbólicas posteriores. Clasificación es un concepto fundamental a este nivel, porque traduce el poder en una estructura simbólica. Desde este punto de vista, cuando hay un fuerte aislamiento entre grupos sociales en la adquisición de los códigos elaborados, esto constituye una re4ización crítica de la clasificación fuerte. La enmarcación, por otra parte, regula la modalidad de socialización dentro de la clasificación para responder a ella. Puede haber continuidad o discontinuidad en las fuerzas de la clasificación y de la enmarcación en diferentes períodos de tiempo tanto dentro de, como entre contextos socializantes diferentes. Es también importante, tener en cuenta que las fuerzas de la clasificación y de la enmarcación pueden variar independientemente una de otra, de tal forma que en un principio se podría tener una agencia regulada por clasificación débil pero con una enmarcación fuerte. Si tal forma de transmisión se puede reproducir exitosamente a si misma, asegurarla que sólo una estructura de inter-relaciones fuera permitida. A un nivel más alto de abstracción tendríamos la paradoja de una clasificación débil que genera una fuerte clasificación. En términos mas específicos, tal forma de transmisión (clasificación débil) de acuerdo con la teoría, descansaría sobre una ideología explícita (la cual reduciría los aislamientos y establecería nuevas interrelaciones), sin embargo, la fuerza de la enmarcación restringiría las opciones disponibles tanto al socializador como al socializado. 
A nivel de transmisión estos conceptos podrían usarse no sólo para el análisis de las agencias de preparación (familia, escuela) y de las agencias de reparación (prisiones, hospitales) sino también para el análisis de las agencias que regulan la transmisión de sistemas simbólicos cruciales para la reproducción cultural. Sin embargo, en los volúmenes 1 y 3 , el análisis está limitado a la familia y a la educación. Así, una de las formas de reproducción de las relaciones de clase se realiza mediante la clasificación fuerte de los códigos elaborados producidos a través de las relaciones de clase que actúan tanto en la familia como en la escuela. Es importante, sin embargo, añadir que las clases no sólo regulan la distribución de los códigos elaborados, sino que también regulan sus manifestaciones.

\section{Nivel III - Textual}

Este aspecto se refiere a la producción de textos específicos en las cuatro situaciones anteriores (instruccional, regulativa, imaginativa e interpersonal). El nivel textual plantea en términos muy rudimentarios los criterios para distinguir, en el nivel semántico, si el texto se realiza a través de una variante elaborada o restringida. Con el propósito de plantear el concepto de código, al nivel empírico, es necesario examinar el tipo de variante en las cuatro situaciones. No es aquí el lugar para discutir las especificidades del análisis sociolingüístico que se puede derivar de esta formulación inicial. Sólo podemos remitir al lector a los ensayos del volumen dos y en particular a la monografía editada por Diana Adlam, Codes in Context (RKP) y a la monografía Familiar Transmission and Socialization.

Debo enfatizar que la distinción entre niveles es analítica. Es claro que, de acuerdo con la tesis, los aspectos (1) y (2) están invisiblemente presentes en el nivel III. Debe enfatizarse que el cambio de los elementos de una situación puede cambiar su manifestación semántica y lingüística. Esto se deduce de la tesis, sin embargo, la producción de una variante elaborada no indica en sí misma que el código haya sido cambiado. Así, la sola exposición a una relación pedagógica de tipo C/F débiles es un indicativo de que el código agregado básico no ha sido adquirido. Si el código subordinante es el elaborado entonces las variantes entran en relación con el código en términos metafóricos de figura y fondo. En este sentido una variante elaborada de un código restringido es diferente de la variante elaborada de un código elaborado. En la misma forma las manifestaciones de las variantes restringidas varían de acuerdo con el código subordinante. Existe una relación dialéctica entre el código de intimidad y el código de distancia-miento, entre las subjetividades locales y la elaboración objetiva de sus bases y condiciones. Esto es equivalente a decir que las relaciones de clase regulan la distribución y manifestaciones de los códigos elaborados pero únicamente las manifestaciones de los códigos restringidos. En otras palabras, todos los individuos poseen un código restringido, pero la significación de sus manifestaciones varía de acuerdo a si éste es el código dominante.

\section{Las clases y la adquisición de los códigos educativos}

La apropiación de los medios educativos articula la socialización efectiva dentro de los mensajes dobles que subyacen y que se manifiestan en sus códigos de transmisión. Desde esta perspectiva podemos alcanzar una comprensión de los orígenes y reproducción de los códigos sociolingüísticos elaborados y restringidos en la familia. En otros términos, de acuerdo con la teoría, los códigos restringidos manifiestan principios y significados dependientes del contexto. Sus principios y significados están ligados a contextos locales, a relaciones sociales, prácticas y actividades locales. Hasta cierto punto 
dichos principios están fuertemente relacionados con una base material específica. Los códigos elaborados manifiestan significados independientes del contexto mediante principios explícitos. Estos principios y significados están menos ligados a un contexto local, o a relaciones sociales, prácticas y actividades locales. Hasta cierto punto los principios y significados están menos relacionados, o relacionados sólo indirectamente, con una base material específica. Ahora bien, ya que estos principios y significados están relacionados sólo indirectamente con una base material específica, se convierten en objeto de especial significación en el sentido estricto, y se convierten en medios de identidad, relevancia y logro individual.

Si ahora miramos las relaciones hipotéticas entre los códigos sociolingüísticos de la familia y la clase social, tenemos, entonces, que los niños de clase media recibirán una transmisión donde las codificaciones tienen una relación indirecta con una base material específica como consecuencia de las relaciones estructurales de la familia con el modo de producción, y de la experiencia de los padres con códigos educativos regulados por las clases sociales. Estos niños serían más sensibles a la incorporación, crucial-mente relevante, de principios para construir textos que están relacionados sólo indirectamente con una base material específica. Además, dichos niños también se volverían sensibles a las relaciones sociales que inducen, demandan y regulan tales realizaciones, manifestaciones, codificaciones y textos, tanto en la familia como en la escuela. Debemos indicar además que el código sociolingüístico de la familia orienta al niño hacia la relevancia de las relaciones sistemáticas entre educación y producción, $y$ hacia las relaciones clasificatorias entre educación y producción, esto es, tanto a la relación de dependencia como a la relación de relativa autonomía entre educación y producción. Las relaciones sistemáticas se traducen en la motivación del niño hacia la educación, independientemente de la relevancia inmediata de ésta y del nivel de actuación del niño. La relación de relativa autonomía se traduce en la orientación del niño hacia los significados independientes del contexto de los códigos educativos y hacia las relaciones sociales que constituyen y regulan la transmisión de sus textos específicos. La clase social crea una correspondencia fundamental entre los códigos educativos y los códigos familiares.

En el caso del niño de clase obrera, podríamos argumentar que las manifestaciones de la transmisión están más directamente relacionadas con una base material como consecuencia de la relación estructural de la familia con el modo de producción y de la experiencia educativa de los padres. Probablemente, estos niños llegarían a ser más sensibles a la incorporación, inicialmente relevante, de principios y significados más directamente relacionados con una base material específica, esto es, serían más sensibles a un código restringido. Sin embargo, tal como lo hemos planteado repetidamente, el código educativo, su forma y contenido, sus reglas de secuencia, ritmo y contenido pueden invalidar y a menudo invalidan la experiencia y la conciencia que los niños traen a la escuela. La escuela descontinúa la experiencia del niño, lo cual está seguido por la descontinuación que el niño hace de la experiencia escolar. Puede suceder que el mensaje dominante del código educativo para los niños o alumnos de clase obrera baja, en relación con los niños o alumnos de clase media, sea aquel constituido por los rasgos clasificatorios internos del código y no por su enmarcación, esto es, por el rasgo pedagógico específico. Si este es el caso, entonces, el niño de clase obrera no es capaz de apropiar los medios para producir lo que cuenta como textos legítimos, aunque es hecho consciente de las relaciones de poder que sostienen los rasgos clasificatorios, y de su lugar dentro de ellos. Aquí, podemos identificar una gran fuerza de resistencia y oposición de parte de tales alumnos. Estas hipótesis pueden ayudamos a entender algunos de los resultados de la investigación de la Unidad de Investigación Sociológica (SRU). 
1) Si consideramos los tests verbales de conciente intelectual $(\mathrm{Cl})$ observamos que los niños de clase media se orientan a ofrecer significados independientes del contexto en ese contexto, y obtienen un puntaje alto; los niños de la clase obrera baja, los cuales se orientan hacia significados dependientes del contexto, obtienen un puntaje bajo.

2) Cuando se solicitó a los niños en un contexto experimental formal que describieran, explicaran o dieran alguna instrucción, nuestra investigación mostró que cuando controlamos el experimento para obtener el $\mathrm{Cl}$ verbal, fue mucho más probable que los niños de 7 años de edad de clase media en relación con niños de clase obrera baja de la misma edad, produjeran textos que manifestaban significados independientes del contexto.

3) En una situación en la cual niños de 8 años de edad tenían que agrupar fotografías de alimentos comunes a ambas clases sociales, los niños de clase medía agruparon los alimentos de acuerdo con un principio taxonómico, y los niños de clase obrera baja los agruparon de acuerdo con sus relaciones diarias (huevos y jamón, deditos de pescado y papitas). El experimento fue diseñado en tal forma que estimulara a los niños a variar el principio de agrupación, y un cierto número de niños de clase obrera baja efectuó el cambio hacia el principio taxonómico.

Ahora bien, cuando analizamos las instrucciones dadas a los niños, podemos notar que éstas son a menudo muy generales y abiertas, como por ejemplo "¿Qué sucede en el cuadro?". "¿Puedes decirle a alguien cómo jugar " $X$ ", quien no sabe como jugar"?. ¿Puedes juntar las figuras en la forma que tú consideres que deben ir juntas?”. "¿Puedes usar todas las figuras o sólo algunas de ellas?". En el lenguaje que hemos usado aquí, la instrucción aparentemente se regula mediante una clasificación y una enmarcación débiles. Lo que parece suceder es algo como esto:

El niño de clase media no acepta la instrucción en términos de la elicitación de una respuesta regulada por -C -F ('Hazlo en la forma que desees' (-C) y 'habla acerca de eso en la forma que tú quieras' (-F). Por el contrario, el niño lo interpreta como si se pidiera una respuesta regulada por \pm C + Fs ("Hazlo en una sola forma y habla acerca de eso en una forma particular"). Así, cuando el niño o la niña están hablando acerca de los cuadros no usan una forma narrativa sino un discurso explícito para describir, a menudo muy cuidadosamente, lo que ellos toman como el rasgo objetivo del cuadro de acuerdo con criterios de verdad o falsedad. El niño no intenta relacionar los detalles del cuadro con su propia vida, su práctica o sus actividades. Cuando estos niños dan las reglas de un juego, muy probablemente, y a diferencia de los niños de la clase obrera baja, intentan dar las reglas abstractas del juego independientes de un contexto local. En la misma forma, los niños de clase media, en relación con los niños de clase obrera baja, agruparon los alimentos de acuerdo con los principios de una taxonomía, antes que de acuerdo con asociaciones de comidas de varias clases. Nosotros creemos que lo que estamos presenciando en este caso tiene muy poco que ver con la "medida de la inteligencia" de los niños, y es, más bien, el resultado de un código comunicativo en el cual las realizaciones simbólicas están relacionadas indirectamente con una base material, lo cual orienta al niño en ciertos contextos hacia la selección de reglas de actuación que crean un texto independiente del contexto. En otras palabras, el niño de clase media es consciente que el adulto no requiere la respuesta que la instrucción a nivel superficial aparentemente demanda. El niño de clase obrera baja, por otra parte, interpreta la instrucción en sus valores superficiales $(-\mathrm{C}-\mathrm{F})$ y de esta manera crea un texto dependiente del contexto. Si 
esta explicación es correcta, nos muestra también cómo las relaciones de clase penetran las transmisiones en la familia aunque nunca en una forma definitiva o irrevocable. Más aún, nos muestra las bases sociales de lo que a menudo se considera como "cogniciones abstractas". Y en la medida en que la explicación plantea estos aspectos, apunta hacia el análisis de sus elementos potenciales y de su regulación por relaciones de clase.

Finalmente, como lo hemos señalado en alguna otra parte, las reglas de secuencia de las transmisiones educativas, el ritmo de la transmisión (la velocidad de adquisición esperada) su futura relevancia o su inmediata irrelevancia, se basan en reglas de actuación que el niño de clase media posee embrionariamente. La clase social regula los códigos elaborados de la educación y los códigos elaborados en la familia. Sin embargo, si comenzamos a partir de la visión de que todos los niños poseen una competencia común, y tratamos de descubrir el mundo que la controla, podemos llegar a entender las bases sociales de las diferencias en la actuación de los niños, y cómo estas actuaciones se constituyen socialmente; podemos llegar a entender cómo y por qué algunas actuaciones se legitiman y otros no, lo cual puede, a su vez crear las condiciones para el cambio, al menos en la educación. 